\title{
PreDarwinian evolution, prebiotic change: a principle of least selection
}

Michael Yarus

Department of Molecular, Cellular and Developmental Biology

University of Colorado Boulder

Boulder, CO 80309-0347

Keywords: RNA world, mean, dispersion, standard deviation, Normal distribution

Mobile: (303) 618-8017

Office: (303) 492-8376

Email: yarus@colorado.edu

\begin{abstract}
Evolution prior to its Darwinian era occurred in an age of variation. Events allowed by starting materials, chemistry and physics were realized, directed by intrinsic probabilities. In this landscape, a principle of least selection defines the most probable evolutionary path. For selection from a population where $x$ varies, the rate of $x$ evolution $d x / d t=\alpha \Delta \mu$. $\mu$ is the population mean of the selected property $x, \alpha$ a proportionality constant. Explicit equations for selected change $\Delta \mu$ are presented. Simple results exist for common cases; for example, selecting for $x \geq$ a threshold. Maximizing $\Delta \mu$ minimizes need for selection. Least selections for $x$ demand maximal dependence on $x$, maximal $x$ standard deviation $\sigma$, and maximal lower $x$ threshold (consistent with the need for a survivor). Least selection is free of the cost of natural selection, thus can support rapid evolution. Least selection also unites previous seemingly independent optima. As examples, evolution via 'starting bloc selection' and 'chance utility' (where selection spans a minimal change) and 'near-ideal reactions' (where variable reactant supplies recurrently create easy reactions) occur via transitory paths of least selection. 'Bayesian convergence' is guaranteed by least selection's extremum (which sharply defines evolution's path). 'Distribution fitness' (specific outcomes selected among multiple concurrent variations) is least selection of rare functional minorities. Inheritance of encoded chemical capabilities first evolves because templating and catalysis are related, potentially joined in a single molecular complex. Least selection defines directions, biostructures define destinations for likely pathways of preDarwinian evolution.
\end{abstract}

\section{Introduction}

Carl Woese (Woese 2002) envisioned extensive cellular evolution before the "Darwinian Threshold", when inheritance appeared (Yarus 2011), allowing later more complex cells to transfer genetic information. Below, we investigate pre-threshold, pre-genetic events. Such an archaic Earth is a chemical world, hosting undirected, unencoded reactions. Nevertheless, evolution in such a landscape has predictable qualities. Primordial progress toward organisms 
that metabolize, inherit, and perhaps approach the Darwinian threshold, may therefore be intelligible.

Consider the complexity that must be captured by any such theory. In Fig. 1, a range of chemical products expands due to reactions of natural chemical starting materials (at a). The double-dotted line surrounds an area representing accessible variation accessible from natural starting materials. These materials are modified by interaction with other aspects of the environment, yielding varied structures (blue forms, Fig. 1). Blue potential evolutionary precursors are varied, and varying groups differ in quantity and in utility for further evolution.

Within the accessible chemical range are products which more and less resemble a usable biological advance (green arrow, Fig. 1). Such biologically useful entities are infrequent and do not accumulate within the likely product range. They need not even exist continuously: for example, a nM particle infrequently appears even once in cellular volumes (empty stippled areas, Fig. 1).

Thus evolution readily takes a subtle path. Less abundant structures, less related to starting materials, can still provide the preferred evolutionary transition because a population includes a fortunate subset that satisfies a proto-biological need (near b, Fig. 1). Moreover, forms known to laboratory experiments (red outlines, Fig. 1) are unlikely to completely sample possible conformations or chemistry. A conceptual barrier to study of prebiological evolution is that even the simplest replication, metabolism and expression are complex molecular events (blue forms, Fig. 1) - thus, there will be no obvious primordial route to them. Instead, research must identify the least improbable routes to biological complexities.

This process is described in sections meant to be semi-independently readable: first analysis, then numerical illustrations, then previously analyzed examples. Putting Fig. 1's implications in explicit form begins with selection.

Effective change in a distributed population. Selection for $x$ is represented as:

$$
\varepsilon=a+b x
$$

where $\varepsilon$ is fold increase due to a selective evolutionary episode. Within $\varepsilon, a$ is increase independent of condition or molecule $x$. Increase dependent on the advantageous condition is $b x$, with increase proportional to the amount of favorable $x$. $\varepsilon$ might be a probability, and $0 \leq \varepsilon$ $\leq 1$. Alternatively, $\varepsilon$ can measure general response to selection; for favored $x, \varepsilon$ is then any positive real number, as is the case below.

The probability of $x$ is described by a normalized probability distribution function, $p d f$ :

$$
\int_{-\infty}^{\infty} p d f(x) d x=1
$$

To follow the fate of a population, its average $x$ is followed here. If $p d f(x)$ is the probability of $x$, the average $x$ is $\mu$ : 


$$
\mu=\int_{-\infty}^{\infty} x p d f(x) d x
$$

Selection increases $x$ :

$$
p d f(x) \rightarrow \varepsilon p d f(x)=(a+b x) p d f(x)
$$

Alternatively, evolution may propagate favored individuals already in the population. Most flexibly, greater $x$ may evolve by both selective increase as well as acceptance of individuals having $x$ greater than a threshold, $x_{t}$. For this most general selection, both selection and threshold effects must be normalized. Given that:

$$
\int_{x_{t}}^{\infty}(a+b x) p d f(x) d x=a \int_{x_{t}}^{\infty} p d f(x) d x+b \int_{x_{t}}^{\infty} x p d f(x) d x
$$

where $p d f^{\prime} s$ are those for the starting distribution, the Normal distribution is a natural choice.

Effect on the average of a favored $\boldsymbol{x}$. The Normal, or Gaussian, distribution (Methods) has a special status documented by the Central Limit Theorem (Feller, William 1970). The theorem shows that any property which is the sum of random variables, which may individually be distributed in any way, nevertheless approaches a Normal distribution as the number of variables increase. Normal and near-Normal behavior is accordingly widespread in real-world systems, making it a cogent example. Moreover, because of the broad usage of the Normal pdf, integrals in Eqn 5 are readily evaluated in standard mathematical or spreadsheet software. For example,

$$
\int_{x_{t}}^{\infty} p d f(x) d x=\Phi\left(x_{t}\right)
$$

where $\Phi\left(x_{t}\right)$ is the fraction of the Normal distribution with $x$ between $\mathrm{x}_{\mathrm{t}}$ and $\infty$ (Methods).

The rightward term in Eqn 5 is of intrinsic interest, and can be evaluated by rearrangement:

$$
\int_{x_{t}}^{\infty} x p d f(x) d x=\Phi\left(x_{t}\right) \mu_{s e l}=\int_{x_{t}}^{\infty} \mu p d f(x) d x+\sigma^{2} \int_{x_{t}}^{\infty}(x-\mu) / \sigma^{2} p d f(x) d x
$$

where $\Phi\left(x_{t}\right)$ is the normalizing factor for the integral so that their quotient is $\mu_{\text {sel }}$, the mean of $x$ values above $x_{t}$. The rearrangement on the right can be integrated, because the second term integrates the derivative of the Normal $p d f(x)$ :

$$
\begin{gathered}
\Phi\left(x_{t}\right) \mu_{s e l}=\mu \Phi\left(x_{t}\right)-\sigma^{2}\left[p d f(\infty)-p d f\left(x_{t}\right)\right] \\
\Phi\left(x_{t}\right) \mu_{s e l}=\Phi\left(x_{t}\right) \mu+\sigma^{2} p d f\left(x_{t}\right)
\end{gathered}
$$

The probability of infinite $x, p d f(\infty)=0$. So, mean $x$ above a threshold $x_{t}$ can be expressed as a function of the mean and standard deviation, $\mu$ and $\sigma$, of the initial population placed under selection for $x$, the probability of the selection threshold, $p d f\left(x_{t}\right)$, and the cumulative probability of being above the threshold, $\Phi\left(x_{t}\right)$. The selection integral can now be normalized:

$$
\int_{x_{t}}^{\infty}(a+b x) p d f(x) d x=a \Phi\left(x_{t}\right)+b\left[\Phi\left(x_{t}\right) \mu+\sigma^{2} p d f\left(x_{t}\right)\right]
$$


And:

$$
\int_{x_{t}}^{\infty} \frac{(a+b x) p d f(x) d x}{(a+b \mu) \Phi\left(x_{t}\right)+b \sigma^{2} p d f\left(x_{t}\right)}=1
$$

These results define a new normalized probability distribution, $p d f_{\text {sel }}(x)$, after $a+b x$ selection for $x$, accepting individuals with $\geq x_{t}$, or possibly: both selecting and exceeding a threshold:

$$
p d f_{\text {sel }}\left(x>x_{t}\right)=(a+b x) p d f\left(x>x_{t}\right) /\left((a+b \mu) \Phi\left(x_{t}\right)+b \sigma^{2} p d f\left(x_{t}\right)\right)
$$

So the mean of a superior population that has also been selected for elevated $x$ is:

$$
\mu_{s e l}=1 /\left((a+b \mu) \Phi\left(x_{t}\right)+b \sigma^{2} p d f\left(x_{t}\right)\right) \int_{x_{t}}^{\infty} x(a+b x) p d f(x) d x
$$

Using techniques similar to those for Eqn. 5:

$$
\mu_{s e l}=\left[\mu+S \sigma^{2}+\sigma^{2} p d f\left(x_{t}\right) / \Phi\left(x_{t}\right)+S x_{t} \sigma^{2} p d f\left(x_{t}\right) / \Phi\left(x_{t}\right)\right] /\left[1+S \sigma^{2} p d f\left(x_{t}\right) / \Phi\left(x_{t}\right)\right]
$$

where $S=b /(a+b \mu)$, a recurring factor embodying selection and normalization.

$\mu_{\text {sel }}\left(a, b, \mu, \sigma, x_{t}\right)$ for complete selection with threshold (Eqn 12) has multiple terms, but is easily automated to deliver mean selected $x$ from properties of the starting distribution. For example, below we discuss selection's reliance on initial variability, expressed as population standard deviation $\sigma$.

Varied simpler cases are interesting in themselves, and cab be derived from $p d f_{\text {sel }}(x)$ and $\mu_{\text {sel }}$ in Eqn 10 and 12.

Choosing $x$ greater than a threshold only. No selection exists if $a=1, b=0$, where $p d f_{\text {sel }}(x)=p d f\left(x>x_{t}\right) / \Phi\left(x_{t}\right)$ and selection of $x_{t}$ and values greater, implies:

$$
\mu_{\text {sel }}=1 / \Phi\left(x_{t}\right) \int_{x_{t}}^{\infty} x p d f\left(x>x_{t}\right) d x=\mu+\sigma^{2} \operatorname{pdf}\left(x_{t}\right) / \Phi\left(x_{t}\right)
$$

Later we will need the average threshold-selected change in $x$ :

$$
\Delta \mu=\left(\mu_{\text {sel }}-\mu\right)=\sigma^{2} \operatorname{pdf}\left(x_{t}\right) / \Phi\left(x_{t}\right)
$$

Selection of $x$ only, no threshold. We envision a distribution of $x$ with $\mu>0, \mu$ far enough from $x=0$ so that $p d f(0) \cong 0$. Only positive $x$ are physically meaningful, so $x_{t}=0$ and $\Phi(0)=1$. Under these conditions, $p d f_{\text {sel }}(x)=(a+b x) p d f(x) /(a+b \mu)$ and the mean after selection is: 


$$
\mu_{\text {sel }}=\int_{0}^{\infty} \frac{x(a+b x) p d f(x)}{a+b \mu} d x=\frac{1}{a+b \mu}\left[a \mu+b \int_{0}^{\infty} x^{2} p d f(x) d x\right]=\frac{a \mu+b \overline{x^{2}}}{a+b \mu}
$$

where $\overline{x^{2}}$ is the expected value of the square of $x$. Using a classic statistical identity, $\overline{x^{2}}=\mu^{2}+$ $\sigma^{2}$, so:

$$
\mu_{\text {sel }}=\mu+S \sigma^{2}
$$

The general selected change in $\mu$ is

$$
\Delta \mu=\left(\mu_{s e l}-\mu\right)=S \sigma^{2}
$$

When selection is absolutely dependent on possession of $x(a=0)$, these results are:

$$
\mu_{\text {sel }}=\mu+\sigma^{2} / \mu
$$

And selected change for complete dependence on $x$ is particularly simple:

$$
\Delta \mu=\left(\mu_{\text {sel }}-\mu\right)=\sigma^{2} / \mu
$$

This common situation is worth emphasis: selection requiring $x$ and $\propto x$ in a complete population increases average $x$ precisely by dispersion $\sigma / \mu$ times $\sigma$, independent of the intensity of selection, $\mathrm{b}$. As might be intuitive, selection completely dependent on $x$ produces greater $x$; the relative selected change is larger by the factor $1+a / b \mu$, thus necessarily $\geq 1$.

This analysis can be used to build an intuitive grasp for selection of a favored property $x$ in a varied population. Because we later summarize varied selections with a single equation, note that selection for $x$ as in Eqn 12-18 can be unified as:

$$
\text { selected mean change }=\mu_{\text {sel }}-\mu=\Delta \mu\left(a, b, \mu, \sigma, x_{t}\right)
$$

Selection depicted. Figure $2 \mathrm{~A}$ exemplifies the effect of selection on an initial Normal distribution. The initial distribution is the solid blue line, and an example selected distribution (multiplied by $\varepsilon=0.1+0.5 x$ and renormalized) is dotted red. A logarithmic plot of distribution probabilities makes the same vertical distance the same factor throughout the plot. The downward red arrow at the top marks the selected, increased mean, $\mu_{\text {sel }}$, from Eqn 15:

$$
\mu_{\text {sel }}=10+\frac{0.5(2)^{2}}{0.1+0.5(10)}=10.3922 .
$$

Mean increase is small, $\approx 3.9 \%$, though $x$ values at the right edge of the plot were initially multiplied more than 10 -fold by selection.

$Z_{t}$ provides a generalized way to express $\boldsymbol{x}_{\boldsymbol{t}} . Z$ values are distances from the mean in units of $\sigma$ :

$$
Z=(x-\mu) / \sigma \equiv(x-\sigma / \sigma) / \sigma
$$


$Z$ can also be negative, measuring distance below the mean (Fig. 2A). Use of $Z$ for position on the distribution simplifies comparisons because Normal distributions all have the same probability at the same $Z$ (Methods). The slightly abstruse identity at right in Eqn 21 shows that it is possible to think of Normal probabilities as governed by either $Z(x, \mu, \sigma)$ or $Z(x, \sigma / \mu, \sigma)$ where dispersion $=\sigma / \mu$. The latter can be more convenient. For example, if $\sigma$ is near-constant: sitting at a given $x$ with given variability $\sigma, Z$ and Normal probabilities vary with dispersion.

Selected changes in a distribution. Fig. 2A shows unselected and selected distributions for $x$. The logarithmic vertical axis clearly shows lesser change near the mean (with no change at $\mu$ ), and resolves the favorable increase in upper tail outcomes. The initial Normal distribution (blue) is symmetrical around $\mu=10$, the mean chosen for the selected property. After selection for $x$, the selected, normalized population is altered, with depressed lower $x$ frequencies on the left, and elevated $x$ frequencies above the mean on the right (required for selected frequencies to sum to 1.0). So $x$-dependent selection's effect partially cancels in a normalized population. Fig. 2A's appearance thus anticipates a major conclusion; the most effective selection for $x$ will be one restricted to individuals above the mean, rightward in Fig. $2 \mathrm{~A}$.

Two kinds of progress. There are two ways to select $x$ : one might exploit a favorable fate for $x$ and the resulting increase of the population $x$ embodied in $\varepsilon=a+b x$. Here this will simply be called "selection" for $x$ (Fig. 2A). An alternative is to require $\mathrm{x} \geq \mathrm{a}$ "threshold" value $x_{t}\left(Z \geq Z_{t}\right.$, Fig. 2A) so that a descendant population's mean $x$ increases. Of course, selected increase of all $x$ (via $\varepsilon$ ) and acceptance of $x \geq x_{t}$ can occur simultaneously; both appear here (Eqn 12, Fig. 2).

Threshold effects on $\boldsymbol{x}$ are greater. In Fig. 2B, Eqn 12 is used (for both selection and threshold) and Eqn 13 (for threshold alone) to compare their effects on mean $x, \Delta \mu$, as in Eqn 14. Fig. 2B uses the same conditions as Eqn 20, so the results agree. At the left (blue diamonds), where the acceptable threshold $x_{t} \sim 0\left(Z_{t} \sim-5\right)$ so there is no threshold effect, overall selection is that calculated by Eqn 15, 20 and shown in Fig. $2 \mathrm{~A}, \Delta \mu=0.3922$. This small $\varepsilon$ effect persists as $x_{t}$ and $Z_{t}$ move rightward; but as the lower threshold approaches the mean, the threshold effect, by favoring elevated $x$ (Fig. 2A), becomes significant, and $\Delta \mu$ increases steeply. Notably, with these values, a threshold $Z_{t} \geq 4.8$ (compare Fig. $2 \mathrm{~B}$ ) will more than double mean $x(\Delta \mu \geq$ $10)$; so substantial selected increases are attainable via a single threshold selection.

Red triangles in Fig. 2B represent threshold selection alone (Eqn 13, 14), with no $\varepsilon$ effects. Thus, comparison of the two plots in Fig. $2 \mathrm{~B}$ shows that imposing a threshold is much more productive than selectively increasing $x$. Above the mean, nearly the entire increase is due to threshold's focus on elevated $x$.

Selection also weakens above the mean. Predominance of threshold selection is not solely because $\varepsilon$ selection is weaker; selection also decreases in the presence of a threshold, as $x_{t}$ and $Z_{t}$ move rightward. Fig. $2 \mathrm{C}$ plots the added effect due to $\varepsilon$ (the difference between the two curves of Fig. 2B) and also the fraction of total $\Delta \mu$ due to $\varepsilon$ selection. The $\varepsilon$ effect decreases 
absolutely (curve labeled selection), and because the threshold effect increases, the fraction of $\Delta \mu$ due to $\varepsilon$ selection decreases even more rapidly (curve labeled fraction selection).

Because the effects of selection are smaller than those of the threshold (Fig. 2B), thresholds are emphasized below. However, to understand selection's dependence on $x$ sensitivity, or if interested in a different selection model, Eqn 12, 14, 16, 18 and their development can be used to explore such effects.

Effect of $\sigma$ on evolutionary change. Fig. 3A shows how variability in a starting population alters threshold selection, showing $\Delta \mu$ versus $\sigma$, initial standard deviation. While not completely rigorous (compare Eqn 12, Fig. 5 below), to a precision sufficient for intuition, selected increase in $\mu$ linearly increases with $\sigma$, with no selection possible if there is no variability. As multiple lines for increasing $Z_{t}$ show, mean resulting $x$ also increases as the selection threshold rises.

Effect of $\boldsymbol{Z}_{\boldsymbol{t}}$ on evolutionary change. The latter point is clearer in Fig. 3B, where increase in mean $x, \Delta \mu$ is again presented, but versus selection threshold $Z_{t}$ at several constant initial standard deviations $\sigma$. Now, $x$ is significantly increased even at $Z_{t}=0$ because of integration over every larger $x$. However, selection of increased mean $x$ improves almost linearly as the selection threshold increases above the initial mean $\mu$. The linearly increased slope for $\Delta \mu$ with greater $\sigma$ (as in Fig. 3A, Eqn 12) reappears as several lines for different $\sigma$.

The regular increase with increased $\sigma$ in the extrapolate at the left, selected mean increase $\Delta \mu$ at $Z_{t}=0$, can be derived from Eqn 12 . With a selection threshold set at the initial mean:

$$
\Delta \mu @ \mu={ }_{\left.\left\{{ }^{p d f(\mu)} / \Phi(\mu)^{\}}\right\}+S\right) \sigma^{2}} /\left[1+S \sigma^{2}\{d f(\mu) / \Phi(\mu)\}\right]
$$

where $\Phi(\mu)=0.5$ and $\{p d f(\mu) / \Phi(\mu)\}=0.79788$ when $\sigma=1 ;=0.39894$ when $\sigma=2$ and so on, dividing 0.79788 by the desired $\sigma$ (Methods). As portrayed in Fig. 2B and 2C, extrapolate calculation can be informative because selection and threshold effects can be of similar magnitudes just below and around the mean.

Summary. Selected $x$ increases are near linear with initial $x$ standard deviation $\sigma$ (or with $\sigma / \mu$ ) and almost-linear-with-a-small-calculable-extrapolate with $Z_{t}$ above the mean. These straightforward properties make it feasible to picture a specific selection's effect, or to plot a selection adequate to a desired evolutionary transformation.

The cost of least selection. Tails of the Normal distribution decline increasingly steeply (Fig. 2A). Because frequencies of tail individuals are low, the number needed to expect one improved individual above a threshold increases steeply with increased $\mu_{\text {sel }}$. Integrating frequencies of all cases above threshold $Z_{t}$ in Fig. $4 \mathrm{~A}$, the population for one selected individual, $1 / \Phi\left(Z_{t}\right)$, is shown linearly (blue, solid) and on an exponential axis (red, dashed). The 
population surveyed to find one superior survivor (and proportionately more for multiple survivors) increases very non-linearly (blue, Fig. 4A), upward sharply with selection of more extreme $x$.

For example, selection above $\mu+3 \sigma$ (beyond $Z_{t}=3$ ), increases the selected upper population mean $x$ by $\approx 33 \%$ - a population $\approx 740$ is needed to expect one superior survivor. But if $17 \%$ more $x$ were required (to $50 \%$ more $x$ ), a population of $\geq$ a million must be rejected.

This population effect is generalized in Fig. 4B, which shows integrated selected $\Delta \mu$ and population required to achieve it from varied initial variabilities expressed as standard deviation $\sigma$. Similar, substantial improvements (e.g., 50\%) can be found above moderate thresholds in populations with varied standard deviations. However, a decrease in standard deviation of only 3-fold increases the population for one expected survivor (e.g., at $50 \%$ change) more than 5 orders of magnitude (Fig. 4B). Thus, selection of an upper tail for moderate improvement is undemanding. But, if exceptional increases are vital for evolutionary success - much larger populations, unusual properties, low survivals, and/or elevated variabilities $(\sigma$ or $\sigma / \mu)$ are required. This constraint can be rigorous, with near-exponential increases in numbers required for small improvements in selected change. Accordingly, finely divided populations with small individuals, or unusually varied populations (or both) were surely crucial in the most demanding episodes of chemical evolution. This is an analytical reason to expect microbes emergent at the origin of biology.

Chemical selection is not burdened by the cost of natural selection. The cost discussion above has a distant resemblance to the classical discussion of the cost of natural selection, due to JBS Haldane (Haldane 1957). However, the two cases differ greatly. Haldane's cost was due to replacement of genes in a genetic population: possessors of an old gene configuration must perish to be replaced by a new genetic constellation. Too much replacement yields too much perishing, and thus extinction. Haldane's cost may be mitigated by recombination (Hickey and Golding 2019), so may help account for the prevalence of sexual reproduction. Nevertheless, the rate of natural selection is limited. However, in a non-genetic population, any fraction of a population may be replaced in a short time as long as a small selected portion persists. Thus, preDarwinian change may be sporadic, but can be much more rapid than natural selection, as previously emphasized: inheritance itself might emerge in a few days (Yarus 2017).

Rates of phenotypic change. Just above, quite general statements are made about selections for a distributed quantity $x$. These results imply rates of $x$ evolution. Using Eqn 19 for the change in $\mathrm{x}$ due to selection on [pre] for $x$, we have determined increase in $x$ per selection. If we envision selection as taking place in small population sectors or by increments, we can convert to a continuous form:

$$
d x / d \text { sel }=\Delta \mu
$$


Where $d x / d$ sel is the mean increase in individual $x$ by selection, sel. Or, if the total $x$ of the population is what is selected:

$$
d \sum x /_{d \text { sel }}=\Delta \mu[\text { pre }]
$$

where [pre] is the size of the precursor population. This might be the case, perhaps, if total size determines resistance to desiccation. But continuing with selection of individual $x$, assuming a stable world, selections require a predictable time $t$, sel $=\alpha t$ :

$$
d x /_{d t}=\alpha \Delta \mu
$$

In Eqn 24, selected increases in mean population $x$ occur proportional to time, defining a zeroth-order rate $=\alpha \Delta \mu x /$ time $^{-1}$; where the time unit is that of selection/time proportionality, $\alpha$.

The parameter $\alpha$. We know biotic evolution mostly from recent Earth history, chiefly from durable fossils left by relatively modern, multicellular creatures. Such evolution is sporadic, with long periods of relatively slower change interrupted by shorter eras containing mass extinction (Jablonski 1986, 2002). There have been $\geq 7$ major defined extinctions. Hodgskiss et al (Hodgskiss et al. 2019) present isotopic evidence for one such ending the Great Oxidation Event 2 gigayears ago. For the last 540 million years, see Muscente et al (Muscente et al. 2018) for such alternation of stability and catastrophe in the "big five" extinctions (Benton 1995). Ceballos (Ceballos et al. 2020) provide evidence of a current, human-induced catastrophe Calling these "extinctions" may distort their significance to evolution, as these famous quick losses are also followed by re-diversification of surviving organisms (Hull 2015). Thus the evolution we know contains slow change interspersed with eras of more rapid innovation. It is natural to assume that the preDarwinian era was similarly a mixture of stability and volatility.

Stable eras are treated here as having a characteristic frequency of selections per time, $\alpha$ selections per time unit. Assuming an $\alpha$ does not imply ordered selections. Random events occuring at constant mean probability define a rate constant (Yarus 2021a); thus selection at $\alpha$ per time, on average, readily occurs via random events.

Present inferences are bought at the cost of obligatory assumptions, notably stability. The greater environment is assumed constant for the time of this calculation. That is, selection does not vary, and precursor populations are unchanged, to the precision needed. Evolution therefore will require another approach (perhaps $\alpha=\alpha$ (time)) during catastrophies.

A principle of least selection, one. A particularly useful idea arises from Eqn 23 and 24, explicit rate equations for selected change. In varied realistic cases, most rapid evolution for $x$ occurs when $\alpha \Delta \mu$ is maximized. For Eqn 23, defining such a maximum defines a path requiring least selection. Or, with Eqn 24, such an event occurs first, thereby identifying a most probable evolutionary transition. Maximizing $\alpha \Delta \mu$ recognizes multiple evolutionary effects, incorporating 
both threshold and selection, minimizing duration, and evoking the faintly parallel physical principle of least action (Feynman, RP, Leighton, RB, Sands, M 2010). This extremum potentially finds the most likely route to an improbable outcome (Fig. 1). Call it the principle of least selection. Via Eqn 12, 14, 16, 18 and later formulations in Eqn 23-24, least selection allows relative or absolute times for prebiotic change(s) to be estimated from type of selection, thresholds and population data. In particular, [pre] must be large enough to include an enhanced individual. In Discussion, explicit least selections are considered.

\section{Discussion}

Outline. Fluctuation frequently is the crux of progress in prebiotic chemical evolution (Yarus 2013). As a result, it was proposed that prebiotic chemical progress might be predictable from statistical considerations (Yarus 2021a). Here this idea is explored, in that functions of standard deviation $\sigma$, dispersion $\sigma / \mu, x$ threshold $Z_{t}$, and selection itself, $S$, explicitly determine increases in a selected property (Eqn 12, 13, 15, 17).

The results, viewed broadly, are simple. For Normally-distributed $x$, simultaneously applying selection and a threshold for acceptable $x$, selected change is accurately proportional to standard deviation $\sigma$ or dispersion $\sigma / \mu$ (Fig. 3A, 3B). Improvement in a favored property after selection also has a slope increasing $\approx$ proportionately to a threshold for acceptable $x, Z_{t}$ (Fig. $3 \mathrm{~A}, 3 \mathrm{~B}$, Eqn 22). However, $Z_{t}$ dependence has a significant extrapolate at $Z_{t}=0$ (that is, at the mean, $\mu$; Fig. 3B, Eqn 22) because a threshold at the mean implies integration over an entire upper range (Fig. 2A). However, exploiting this unique upper range potential for rapid evolutionary change requires large populations (Fig. 4A, 4B).

A principle of least selection, two. Variability, selection and threshold together determine selection's effect, $\mu_{\text {sel }}-\mu=\Delta \mu\left(a, b, \mu, \sigma, x_{t}\right)$ (Eqn 12, 19). In light of Eqn 14 and 15, the selected mean $\mu_{\text {sel }}$ in Eqn 12 has the following plausible form:

$\mu_{\text {sel }}=\left[\right.$ initial mean + selection $\Delta+$ threshold $\Delta+$ sel $\mid$ thr interaction $\left.* x_{t}\right] /[1+$ sel|thr interaction $]$

This anglicized form of Eqn 12 explains the decline in selection in presence of a threshold (Fig. 2C). Upper tail $x$ increases are eventually dominated by the $x_{t}$ term in which selection is partially cancelled by the denominator. If net threshold plus selection effects are maximized, seeking the fastest rate and the most likely fate for $x$, a path of least selection emerges. Such maximization defines the most probable next evolutionary step (Fig. 1).

Consequences of a rate equation. A mean increase in $x$ of $\alpha \Delta \mu * t \mathrm{x}$ is selected for an individual in time $t$, so $\Delta x=\alpha \Delta \mu \Delta t$. As an explicit example, under the assumption of evolutionary stability, if doubling of initial $x$ is selected, and time to double is $\delta$ :

$$
\delta=\mu / \alpha \Delta \mu
$$


How much change in $x$ is needed to satisfy a hypothetical selection will vary; we expect a unique value in every selection, and for every $x$. Therefore, we adopt doublings for discussion, knowing that varied numbers of doublings will satisfy specific selections.

Evolution with threshold only. If evolution of $x$ happens via a lower acceptance threshold only, then Eqn 14 applies to Eqn 25:

$$
\delta=\mu \Phi\left(Z_{t}\right) /\left\{\sigma^{2} p d f\left(Z_{t}\right)\right\}[1 / \alpha]
$$

Evolutionary doubling of $x$ occurs in time proportional to $\mu$ and the integral $\Phi\left(Z_{t}\right)$. But with constant threshold $Z_{t}, \Phi\left(Z_{t}\right)$ is also constant. Moreover, $\sigma^{2} p d f\left(Z_{t}\right)$ is proportional to $\sigma$ (Methods). This makes it simple to portray the effect of initial variabillity $\sigma$ on threshold acceptance of $x$. Fig. 5 shows that $x$ doubling time $\delta$ is strictly linear in 1/ $\sigma$ (Eqn 26; compare Fig. 3A for mixed selection and threshold). Other factors in Eqn 26 multiply [ $1 / \alpha$ ], the average time for a selection. So Fig. 5 and Eqn 26's time unit is selection times. In addition, inspecting a Fig. 5 column with fixed $\sigma: \delta$ decreases with increased threshold $Z_{t}$, but this is less effective as $Z_{t}$ increases. As noted above, Fig. 5 also shows that with substantial $\sigma$ and substantial $Z_{t}$, very significant change, such as doubling mean $x$ in one selection time, is attainable, though such rapid advances imply rigorous selection in large populations (Fig. 4A, 4B).

Evolution with selection only. Eqn 16 and 25 can be combined in the same way as above to determine doubling time for selection on an entire distribution (no threshold);

$$
\delta=\mu /\left\{\sigma^{2} S\right\}[1 / \alpha]
$$

For example, Eqn 26 and 27 together mean that under the conditions used in these figures (legend, Fig. 2A), at $Z_{t}=2, x \geq$ threshold evolves $\operatorname{pdf}\left(Z_{t}\right) / \Phi\left(Z_{t}\right) /_{S} \cong 12$-fold more quickly than by selection alone. Calculations as in Fig. $3 A$ show that this 12 -fold threshold population increases its mean $x$ by $48 \%$, but it need not be large or finely divided, having $\geq 44$ individuals (Fig. 4A).

Thus, three summary ideas: evolutionary change via threshold is important (compare Fig. 2B) and in real cases, may be the only significant source of selected change in $x$ (Fig. 2C). Moreover, thresholds are the key to the superiority of preDarwinian over natural selection (Haldane 1957); thresholds can yield "jumps" in $x$ without large delays, in one selection time. Lastly, this is an illustration of least selection - evolution via threshold, when it can be used, needs fewer selection times (Eqn 23, 26); and is thus a more likely route to elevated $x$ than selection alone (Eqn 24, 27).

Varied other ideas have numerical consequences, given that selection requires times determined by measurable qualities of a population requiring $x$ (Eqn 12, 13, 15; Fig. 3, 4, 5). 
Prior examples: Starting bloc selection exploits inevitable dispersion. Least selection also conceptually unifies previous effective examples of evolution, clarifying underlying similarities.

The starting bloc (Yarus 2018) was encountered while calculating whether known ribonucleotide reactions suffice for evolution of encoded, and therefore heritable, chemical capacities (Yarus 2017); that is, for the emergence of inheritance itself. The result was that inheritable (bio)chemical reactions are in reach of plausible selection. Heritability's emergence depends on two known RNA capabilities. The first is existence of simple catalytically active RNAs, homologues of the adenylate-dinucleotide-containing enzyme cofactors (White 1976; Yarus 2011). The second is templated synthesis of similar molecules via an ultimately simple, geochemically available (Ferris et al. 1996) RNA "gene", poly-U (Puthenvedu et al. 2015). Selection of such template-directed catalytic synthesis of cofactors (in preference to untemplated chemical synthesis) is uniquely effective early, when only some of potentially capable sites have actually begun synthesis (called the "starting bloc", (Yarus 2018)). Increases of templated synthesis selected in such a population are nearly proportionate to dispersion $(\sigma / \mu)$ in the chemically active product over a large range of product concentrations (Yarus 2018), as anticipated for least selection here (Eqn 21, Fig. 3A, 3B).

Starting bloc selection employs distribution tails. The original starting bloc (Yarus 2018) also produced roughly proportionately faster change when carried out with lower survival after selection, a condition that was called "stringency". This parallels present calculations: moving upward from $\mu$ and downward in survival (increasing $Z_{t}$, Fig. 3B) produces faster selection. Moreover, combining variability (as in dispersion $\sigma / \mu$ or standard deviation $\sigma$ ) with stringency or survival (as in $Z_{t}$ ) in a single index (as in Eqn 19) previously best predicted selection's success (Yarus 2018).

Starting bloc selection exploits a threshold. The difference between functioning pools, which make an active molecule early, and inactive ones with no product is particularly readily selected. Starting bloc success via some, versus no, early activity exploits evolution via a threshold, roughly paralleling least selection in Fig. 2B.

Accordingly, starting bloc selection of a genetically encoded chemical capacity (Yarus 2017, 2018) follows, with almost formulaic precision, the variability, survival and threshold dependence defined for least selection (Fig. 3A, 4A).

Prior examples: Chance utility captures favorable dispersion. Prebiotic systems must evolve toward biological complexity before genes exist, or biology would be inaccessible. Selected temporary, or especially, permanent change in chemical systems before the advent of genetic inheritance has been called 'chance utility'. As an example, variation in a 100 -fold more concentrated inhibitor frequently provided an opportunity to capture an apparently overwhelmed, but favorable, minor reaction (Yarus 2016). Accidently favorable contravariation of a substrate and its competitor relies on means and favorable variations for these reactants; that is, on chance resemblance to a useful result, an opportunity for least selection. 
Prior examples: Random reactant supplies necessarily support near-ideal reactions. Even with random, uncontrolled nucleotide supplies, a specific series of substrates required to favor a hypothetical small RNA replication reaction necessarily recur. But notably, most of the output from such a hypothetical randomly-fed reactor actually comes from a recurrent subset of nearideal substrates and their replication cycles (Yarus 2013). This ability of a randomly-fed pool reactor to produce recurrent specific results from utterly unregulated inputs illustrates the influence of minority near-ideal events, requiring least selection.

The starting bloc, the chance utility example, and the mechanism of near-ideal reactions illustrate a particular theme: optimization can be in the time domain. The occasion for least selection can flicker: briefly appearing, though usually absent.

Prior examples: Bayesian convergence follows from least selection's extremum. Bayes' theorem says that a correct origin hypothesis explains all aspects of a present phenomenon, thereby validating its mechanistic suggestion. However, validation is uniquely effective when independent aspects of a phenomenon are explained, and the hypothesis' probability is elevated by the product of independent confirmations (Yarus et al. 2005). For example, an origin hypothesis for the Standard Genetic Code (Yarus 2021c) explains how metabolic, chemical and wobble order coexist without mutual interference in a near-universal coding table. Bayesian convergence is robustly supported by a principle of least selection, which maintains that the historical route to the genetic code was distinct, perhaps unique, because its route was determined by an extremum in relevant factors (Eqn 12, 24). This idea, in turn, is a specific expression of Orgel's dictum that evolution exhibits Continuity (Orgel 1968); that is, later evolutionary states are necessarily linked to a unique precursor by continuous change.

Prior examples: Distribution fitness embodies evolution's subtlety. The fitness of a superior group within a distribution is a key to apparently improbable outcomes (Fig. 1). For example, a small minority of nascent coding tables has unlikely properties (Yarus 2021a); they quickly become ordered, make accurate Standard Genetic Code assignments (Yarus 2021b), leaving unassigned triplets (Yarus 2021a) that will later be critical during final assembly of a complete code (Yarus 2021c). They short-circuit the outward problem of evolving a complex biological configuration by accidently evolving it simply. This apparently implausible behavior; for example, showing what can seem to be foreknowledge of problems of late coding early in their history, is possible because they are an improbable minority later selected among highly distributed nascent codes. Their properties (Yarus 2013) will ultimately seem near-ideal, but they pre-exist without purpose (Fig. 1). When selected from a varied population because they express the most functional genetic codes (Yarus 2021c), they immortalize their improbable histories, presenting a seeming paradox.

Distribution fitness is least selection, using different language. Distribution fitness was initially devised to clarify rapid, simultaneous progress toward the Standard Genetic Code's complex assignments, chemical order and compact code groupings (Yarus 2021a). Thus, though less 
specific, distribution fitness anticipates least selection - success from rare precursors with close resemblance(s) to a complex biological achievement (Fig. 1).

Least selection can appear as similarity to archaic forms. Finally, least selection can be literal; that is, vast gaps in time and conditions can link two very similar things. Ribosomal peptidyl transferase appears to be such a case. CCdApPuro (Welch et al. 1995) is a rationally-designed inhibitor that binds both halves of the ribosomal active site, contacting the A- and P-sites in a configuration that would allow peptide transfer between them. That is, CCdApPuro emulates the transition state for peptide synthesis. When an RNA was selected from randomized sequences to bind CCdApPuro with affinity similar to ribosomes (Welch et al. 1997), the two most probable binding sites had a 17 nucleotide homology with the peptidyl transferase loop of rRNA. This homology included an exact 8-mer match with among the most conserved RNA sequences known, the ribosomal peptide transferase active site AUAACAGG. CCdApPuro also bound this RNA octamer in the large ribosomal subunit (Ban et al. 2000), in an orientation similar to selected RNA sequences (Welch et al. 1997; Yarus and Welch 2000). Selected ribonucleotides even include a likely catalytic feature, an A with a shifted pKa, though catalysis was not selected (Yarus and Welch 2000). These results strongly support initial peptide synthesis in an RNA cradle, and then least selection to its modern ribonucleoprotein descendant, still easily recognized billions of years later. Peptide transfer bridges preDarwinian and Darwinian eras, hinting that least selection may bear on Darwinian selection also.

Least selection and biological direction: paths and destinations. A small rather than a grand claim is made here; pursuit of least selection will not suddenly manifest prebiotic history. Neutral and near-neutral chemical change (Kimura and Ohta 1971) has not been considered. In a particular case, plausible precursors may not yet be identified (Fig. 1), so a usable selection may not yet be defined, much less quantitated.

Specifically, our present molecular portfolio is critically partial. For example, prebiotic nucleotide activation has been a difficult but essential missing link to both ancient translation and replication. Discovery of a possible prebiotic, UV-activated imidazole activation of AMP (Mariani et al. 2018) has guided related chemistry with 2-amino-imidazole and ensuing templated RNA synthesis with activated CMP and GMP (Zhang et al. 2020). Thus, a vital activation gap may ultimately be filled.

Future research will find further credible structures and suggest effective selections. Least selection at individual steps adds to the previous suggestion that the most plausible pathway yields a biological goal with greatest probability (Yarus 2021a). Assembling least selections to compose a probable path to a well-known biological result winnows feasible possibilities. Ultimately, incomplete or improbable explanations are excluded by more inclusive and plausible ones; a rational route to biology via least selections can come to light (Fig. 1). 


\section{Methods.}

Numerical illustrations. Calculations were executed on a Dell XPS laptop with an Intel Core i98950HK CPU @ 2.90GHz and 32 GB of RAM, running 64-bit Windows 10 Enterprise v. $20 \mathrm{H} 2$. Figures were prepared with Microsoft Excel 2016 16.0.5161.1002, making particular use of the Excel norm.dist function for Normal pdf and cumulative distributions.

As an example, in Eqn 6, $\Phi\left(x_{t}\right)$ is an integral not expressible in closed form using simple functions. But $\Phi\left(x_{t}\right)$ is readily accessible via the widely-available cumulative Normal distribution function $C\left(\mu, \sigma, x_{t}\right)$ (which yields the fraction of the distribution between - $\infty$ and $\left.x_{t}\right): \Phi\left(x_{t}\right)=1$ $C\left(\mu, \sigma, x_{t}\right)$.

The Normal probability distribution function. The Normal pdf used here (e.g., in Eqn 7) is:

$$
p d f(x)=e^{-\frac{1}{2}\left(\frac{x-\mu}{\sigma}\right)^{2}} / \sqrt{2 \pi} \sigma=e^{-\frac{z^{2}}{2}} / \sqrt{2 \pi} \sigma
$$

using the notation defined in the text. The standard deviation $\sigma$ in the denominator is significant because it differs from the Standard Normal distribution, often seen in mathematical references (Greene, William H. 2003). The Standard Normal has a fixed mean and standard deviation, thus is not well suited to thought about their variations. The denominator $\sigma$ just above cancels exponents in (e.g.) Eqn 12, allowing $\Delta \mu$ to vary roughly as does $\sigma$, not as $\sigma^{2}$ (compare Fig. 3A), also causing the extrapolate increase for $Z_{t}$ in Eqn 22, and accounting for linearity in Fig. 5.

The complete $\boldsymbol{\mu}_{\boldsymbol{s e l}}$ selection function. Integration of Eqn 11 to yield Eqn $12, \mu_{\text {sel }}\left(a, b, \mu, \sigma, x_{t}\right)$, requires $\overline{x^{2}}$ for a truncated Normal distribution above $x_{t}$ (that is, integrated $x_{t} \rightarrow \infty$ ):

$$
\overline{x^{2}}=\mu^{2}+\sigma^{2}+\sigma^{2}\left(\mu+x_{t}\right) \operatorname{pdf}\left(x_{t}\right) / \Phi\left(x_{t}\right)
$$

using notation defined in the text. This integration has been omitted for conciseness; a useful introduction to truncated Normal distributions can be found in Greene (Greene, William H. 2003).

\section{Figure legends.}

Figure 1. Ideogram of pre-genetic evolution. Natural primordial materials (arising at a) are transformed into a variety of at least transiently-existing products (blue forms) within the limits of chemical and physical laws (double-dotted line). Some of these forms may be known to modern laboratories (red outlines). A form suitable for advanced biological function arises (at b) and is selected for further evolution in a subsequent pre-biological context (green arrow).

Figure 2A. Selection and threshold acceptance in the Normal distribution. The Normal probability of individual $x, p d f(x)$, is plotted as a solid blue line against a logarithmic ordinate, 
assuming illustrative values: mean $\mu=10$ and standard deviation $\sigma=2$. This distribution is selected by multiplying by $\varepsilon=a+b x$ (dashed red line); $a=0.1, b=0.5$. Labeled arrows show the resulting new selected mean, $\mu_{s e l}$, and blue arrows illustrate the relation between the distributed quantity $x$ and the generalized co-ordinate $Z$ for the initial Normal; $\mu=10, \sigma=2$.

Figure 2B. Selected increase in mean, $\Delta \mu$, across a Normal distribution as the acceptable threshold $Z_{t}$ varies. $Z_{t}$ ranges from far below the mean $\mu\left(Z_{t}=-5\right)$ to an equal distance above $\mu\left(Z_{t}=5\right)$. Blue diamonds are created by selection and threshold accceptance together (Eqn 12), and threshold acceptance alone is shown as red triangles (Eqn 14). Normal and selection parameters are those in Fig. 2A.

Figure 2C. Selection across a distribution that also has a threshold. The difference between the means of the selection+threshold and threshold curves in Fig. 2B is labelled "selection" (red solid line), and the fraction of total difference that is selection, labelled "fraction selection" (blue dashed line)

Figure 3A. Selected increase in mean $x, \Delta \mu$, as a function of increasing standard deviation $\sigma$. Different labeled lines show the effects of selection at varying thresholds, $Z_{t}$, above the mean. Parameters are those of Fig. 2A, which mandate both threshold and selection effects.

Figure 3B. Selected increase in mean $x, \Delta \mu$, as a function of threshold position $Z_{t}$, holding standard deviation $\sigma$ constant at labeled values. Parameters are those of Fig. 2A.

Figure 4A. Population required to expect one selected individual, $1 / \Phi\left(Z_{t}\right)$, as a function of standard deviations above the mean, $Z_{t}$. Populations are plotted both against a logarithmic ordinate (red dashed), and a linear one (sold blue line). Parameters are those of Fig. $2 \mathrm{~A}$.

Figure 4B. Populations required to expect one individual with a selected $\Delta \mu$ at different initial standard deviations $\sigma$. Other parameters are those of Fig. 2A, so the initial mean $\mu=10$.

Figure 5. Evolutionary $x$-doubling times $\delta$ versus initial standard deviation $\sigma$. An evolving population that undergoes only threshold selection of $x$ is assumed (compare Fig. 3A for threshold+selection together), with parameters as in Fig. $2 \mathrm{~A}$.

\section{References.}

Ban N, Nissen P, Hansen J, Moore PB, Steitz TA. 2000. The complete atomic structure of the large ribosomal subunit at 2.4 A resolution. Science 289: 905-20.

Benton MJ. 1995. Diversification and extinction in the history of life. Science 268: 52-58.

Ceballos G, Ehrlich PR, Raven PH. 2020. Vertebrates on the brink as indicators of biological annihilation and the sixth mass extinction. Proc Natl Acad Sci U S A 117: 13596-13602. 
Feller, William. 1970. An introduction to probability theory and its applications, v II, 2nd edition, p. 258-274.

Ferris JP, Hill AR, Liu R, Orgel LE. 1996. Synthesis of long prebiotic oligomers on mineral surfaces. Nature 381: 59-61.

Feynman, RP, Leighton, RB, Sands, M. 2010. The Feynman Lectures on Physics. Millenium Edition. California Institute of Technology.

Greene, William H. 2003. Econometric Analysis; Section 22.2, Truncated DIstributions. 5th ed. Prentice Hall.

Haldane JBS. 1957. The cost of natural selection. J Genet 55: 511.

Hickey DA, Golding GB. 2019. Sex solves Haldane's dilemma. Genome. https://cdnsciencepub.com/doi/abs/10.1139/gen-2019-0051 (Accessed June 16, 2021).

Hodgskiss MSW, Crockford PW, Peng Y, Wing BA, Horner TJ. 2019. A productivity collapse to end Earth's Great Oxidation. Proc Natl Acad Sci U S A 116: 17207-17212.

Hull P. 2015. Life in the Aftermath of Mass Extinctions. Curr Biol CB 25: R941-952.

Jablonski D. 1986. Background and mass extinctions: the alternation of macroevolutionary regimes. Science 231: 129-133.

Jablonski D. 2002. Survival without recovery after mass extinctions. Proc Natl Acad Sci U S A 99: 8139-8144.

Kimura M, Ohta T. 1971. On the rate of molecular evolution. J Mol Evol 1: 1-17.

Mariani A, Russell DA, Javelle T, Sutherland JD. 2018. A Light-Releasable Potentially Prebiotic Nucleotide Activating Agent. J Am Chem Soc 140: 8657-8661.

Muscente AD, Prabhu A, Zhong H, Eleish A, Meyer MB, Fox P, Hazen RM, Knoll AH. 2018. Quantifying ecological impacts of mass extinctions with network analysis of fossil communities. Proc Natl Acad Sci 115: 5217-5222.

Orgel LE. 1968. Evolution of the Genetic Apparatus. J Mol Biol 38: 381-393.

Puthenvedu D, Janas T, Majerfeld I, Illangasekare M, Yarus M. 2015. Poly(U) RNA-templated synthesis of AppA. RNA 21: 1818-25.

Welch M, Chastang J, Yarus M. 1995. An inhibitor of ribosomal peptidyl transferase using transition-state analogy. Biochemistry 34: 385-90.

Welch M, Majerfeld I, Yarus M. 1997. 23S rRNA Similarity from Selection for Peptidyl Transferase Mimicry. Biochemistry 36: 6614-6623. 
White HB. 1976. Coenzymes as Fossils of an Earlier Metabolic State. J Mol Evol 7: 101-104.

Woese CR. 2002. On the evolution of cells. Proc Natl Acad Sci U A 99: 8742-7.

Yarus M. 2013. A ribonucleotide origin for life - fluctuation and near-ideal reactions. Orig Life Evol Biosph 43: 19-30.

Yarus M. 2016. Biochemical Refinement Before Genetics: Chance Utility. J Mol Evol 83: 89-92.

Yarus M. 2017. Efficient Heritable Gene Expression Readily Evolves in RNA Pools. J Mol Evol 84: 236-252.

Yarus M. 2018. Eighty routes to a ribonucleotide world; dispersion and stringency in the decisive selection. RNA 24: 1041-1055.

Yarus M. 2021a. Evolution of the Standard Genetic Code. J Mol Evol 89: 19-44.

Yarus M. 2011. Getting past the RNA world: the initial Darwinian ancestor. Cold Spring Harb Perspect Biol 3, 10.1101/cshperspect.a003590.

Yarus M. 2021b. Optimal Evolution of the Standard Genetic Code. J Mol Evol 89: 45-49.

Yarus M. 2021c. Packing the Standard Genetic Code in its box: 3-dimensional late Crick wobble. BioRxiv 2021.01.18.427168.

Yarus M, Caporaso JG, Knight R. 2005. Origins of the genetic code: the escaped triplet theory. Annu Rev Biochem 74: 179-198.

Yarus M, Welch M. 2000. Peptidyl transferase: ancient and exiguous. Chem Biol 7: R187-190.

Zhang SJ, Duzdevich D, Szostak JW. 2020. Potentially Prebiotic Activation Chemistry Compatible with Nonenzymatic RNA Copying. J Am Chem Soc 142: 14810-14813. 
bioRxiv preprint doi: https://doi.org/10.1101/2021.06.27.450095; this version posted July 7, 2021. The copyright holder for this preprint (which was not certified by peer review) is the author/funder, who has granted bioRxiv a license to display the preprint in perpetuity. It is made available under aCC-BY-NC-ND 4.0 International license.

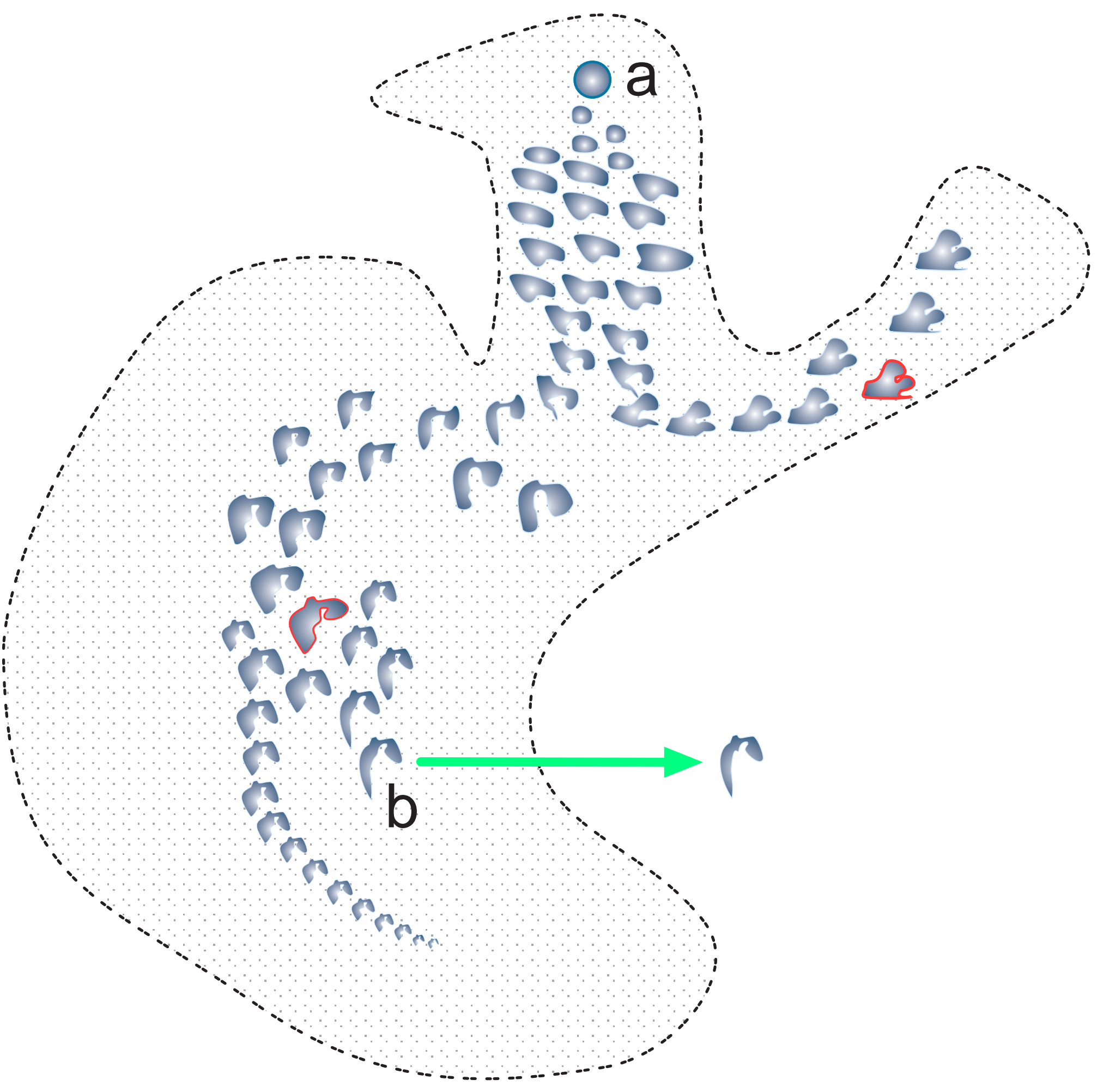




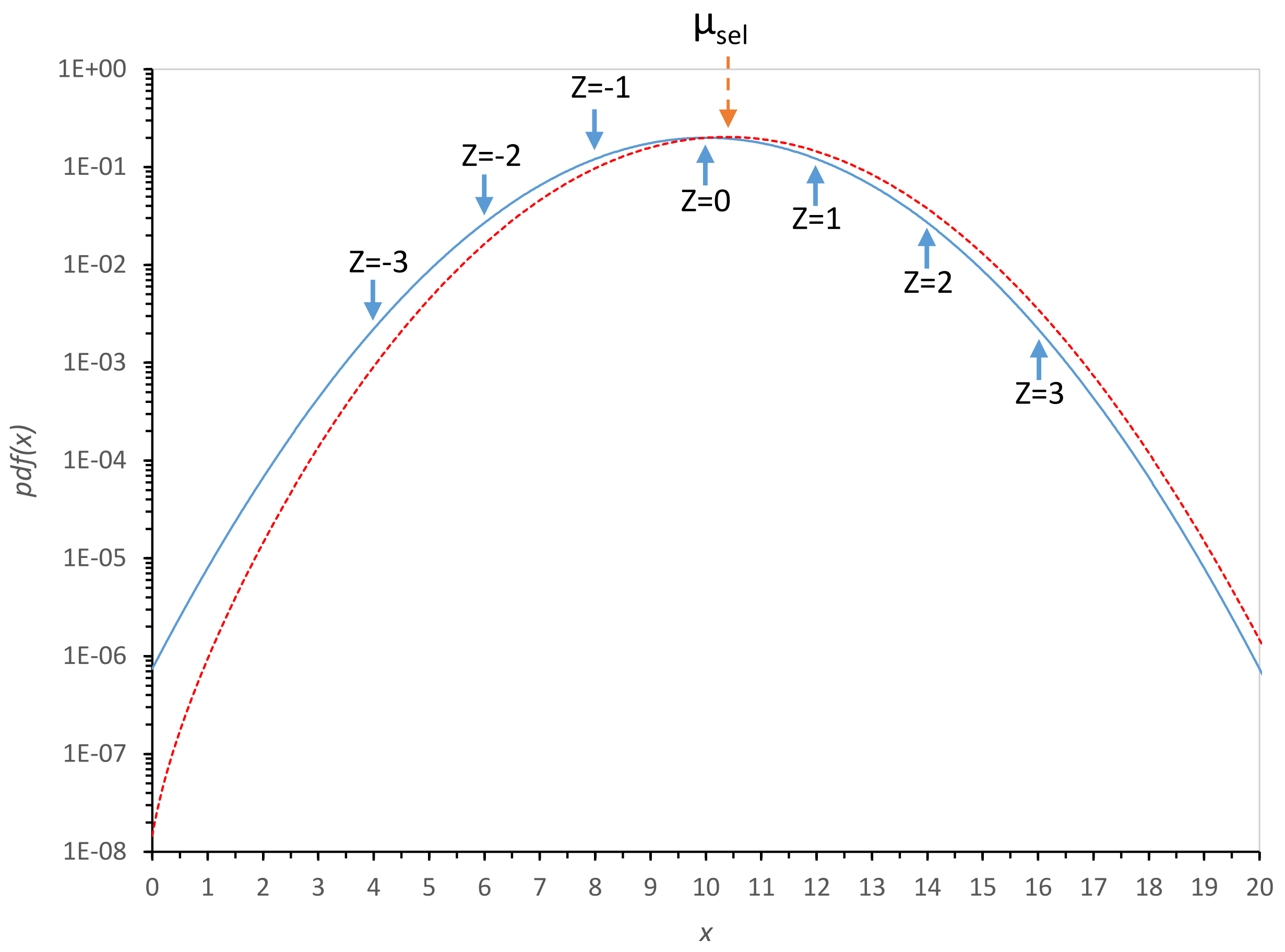

Figure 2A 


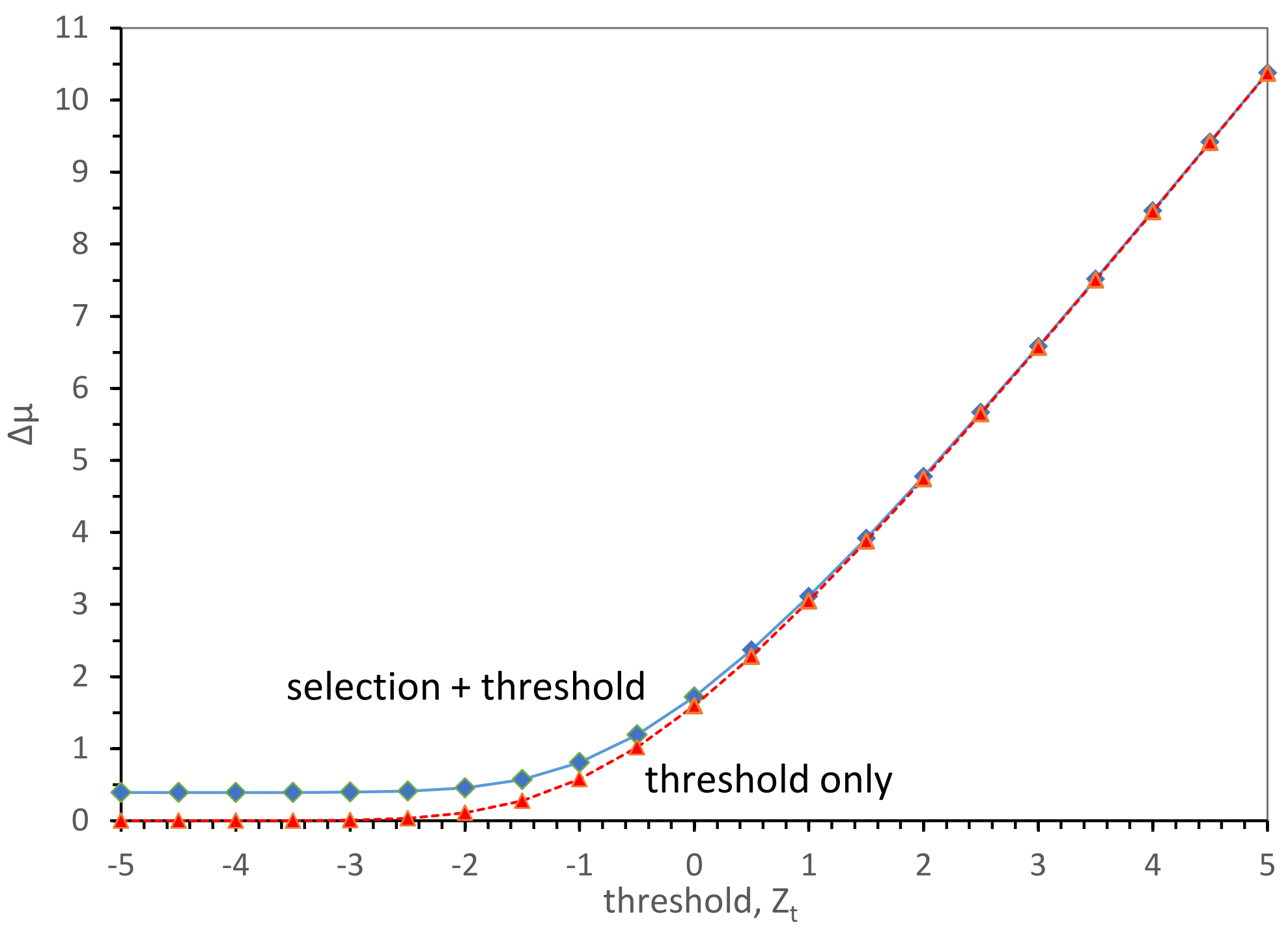

Figure 2B 


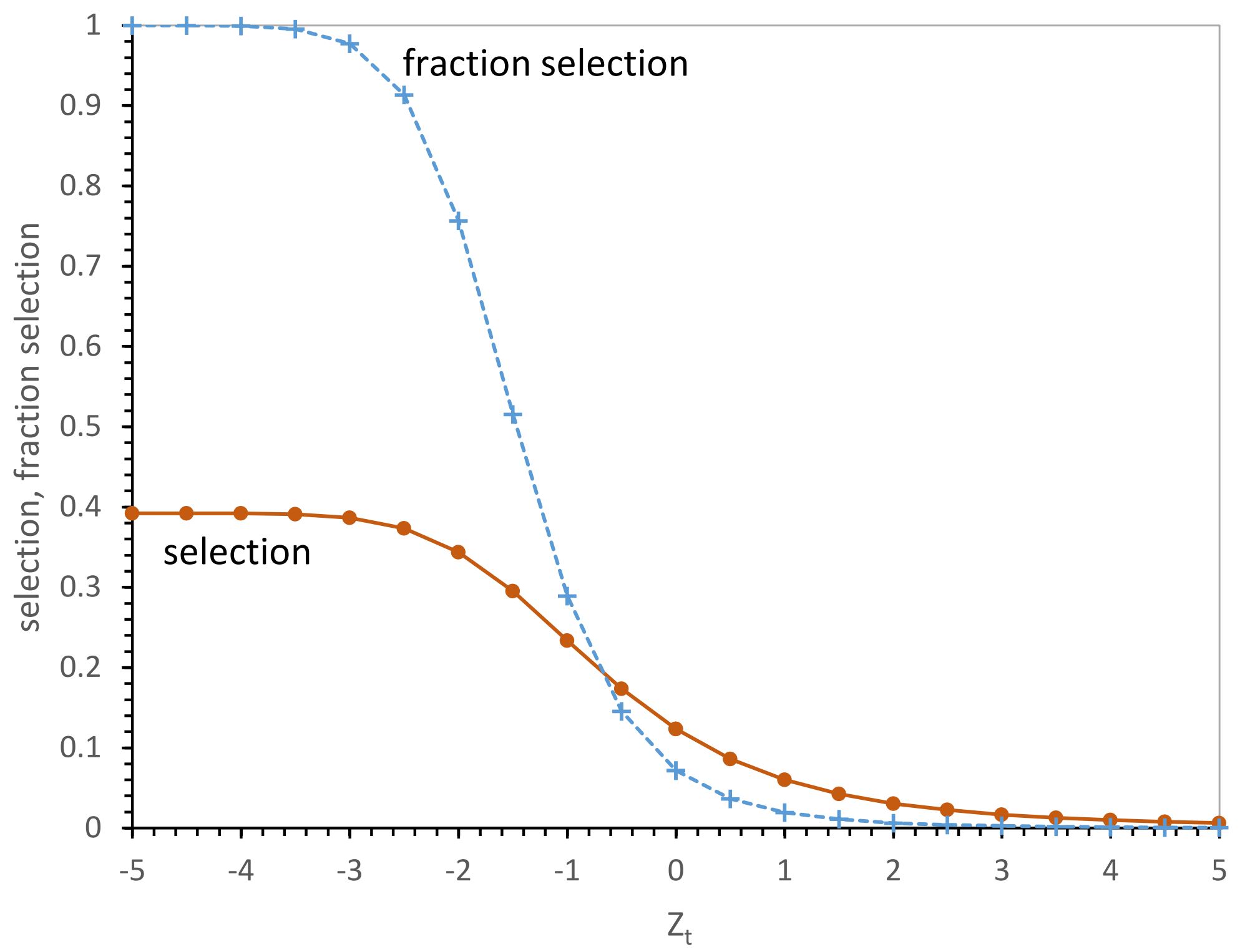

Figure 2C 


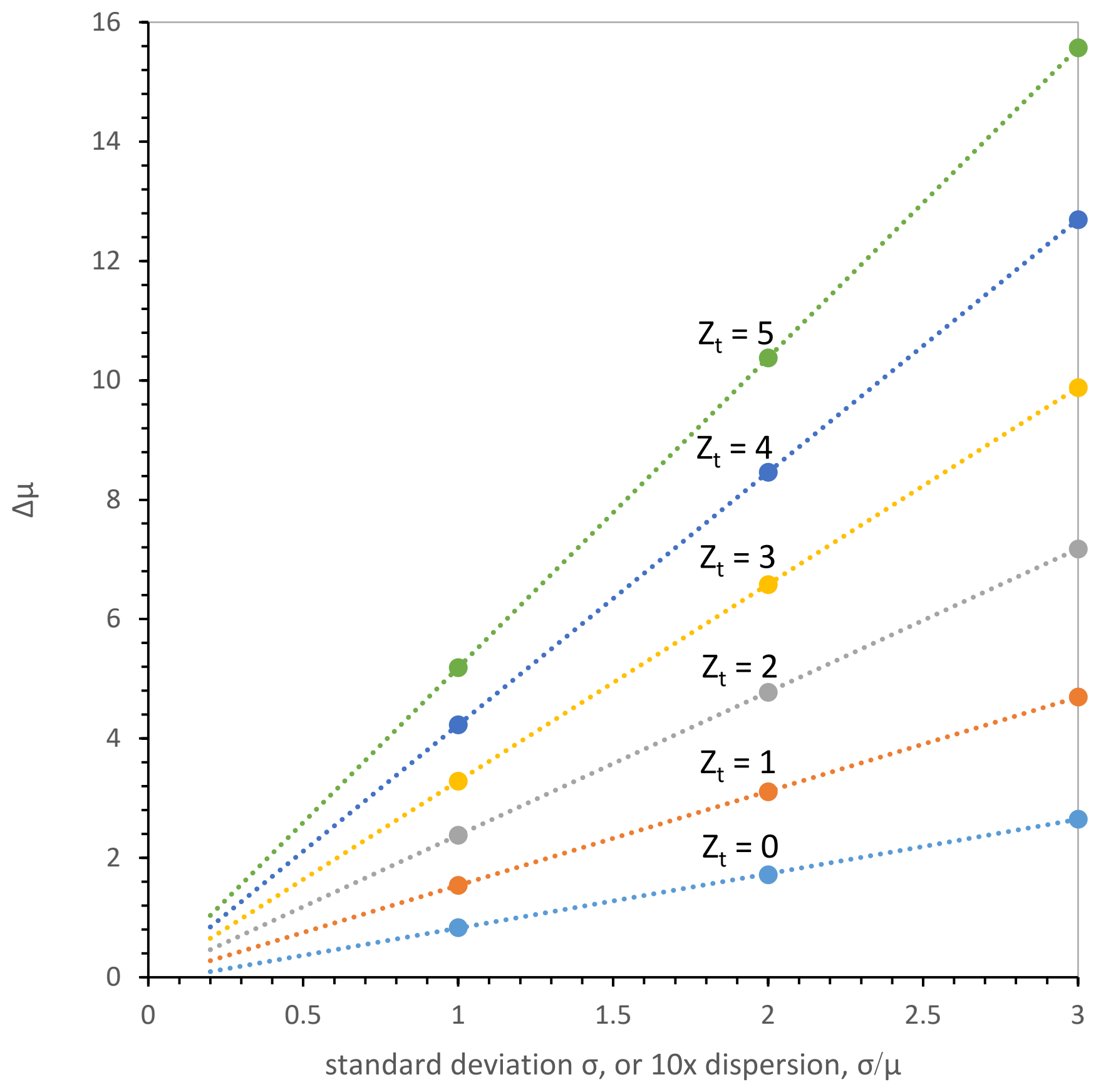

Figure $3 \mathrm{~A}$ 


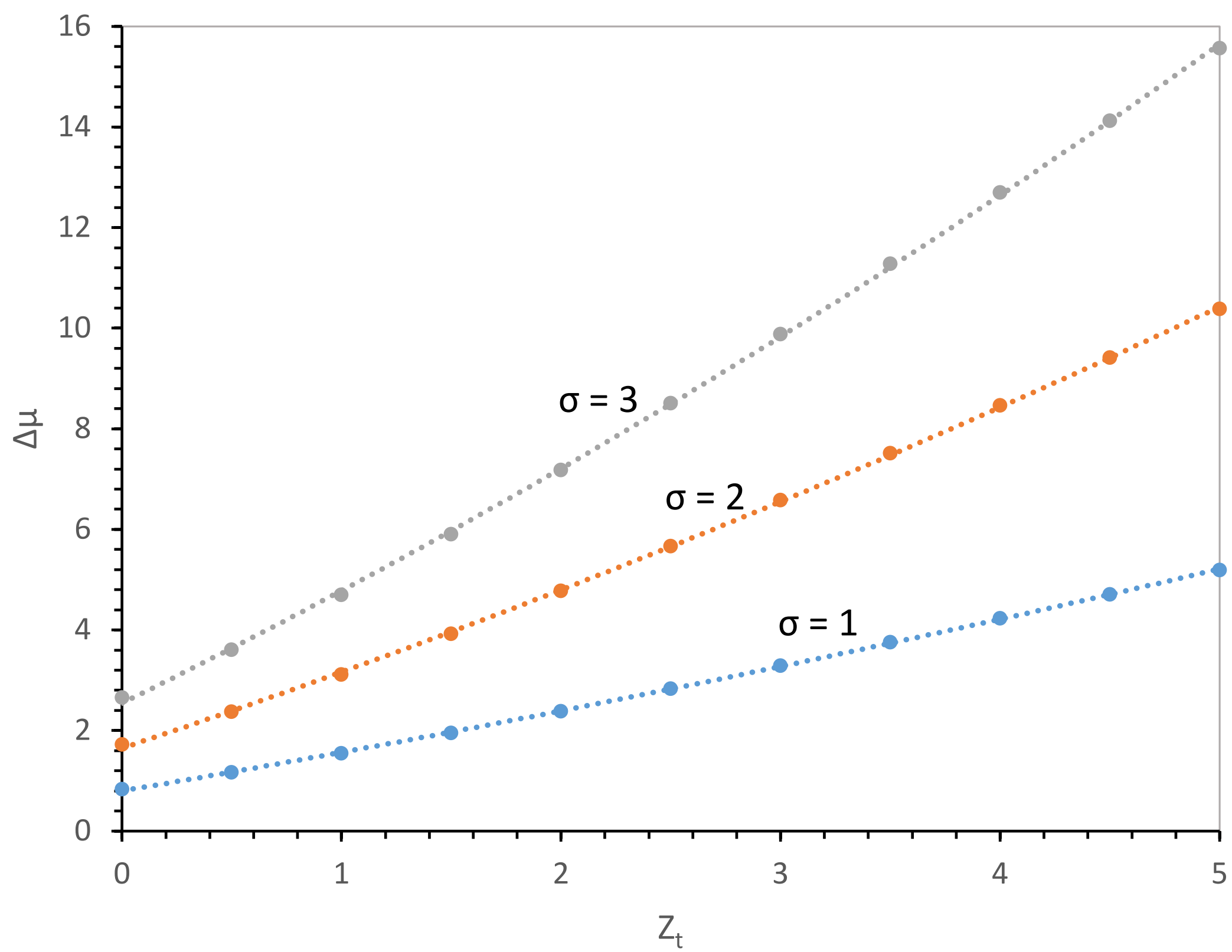

Figure 3B 


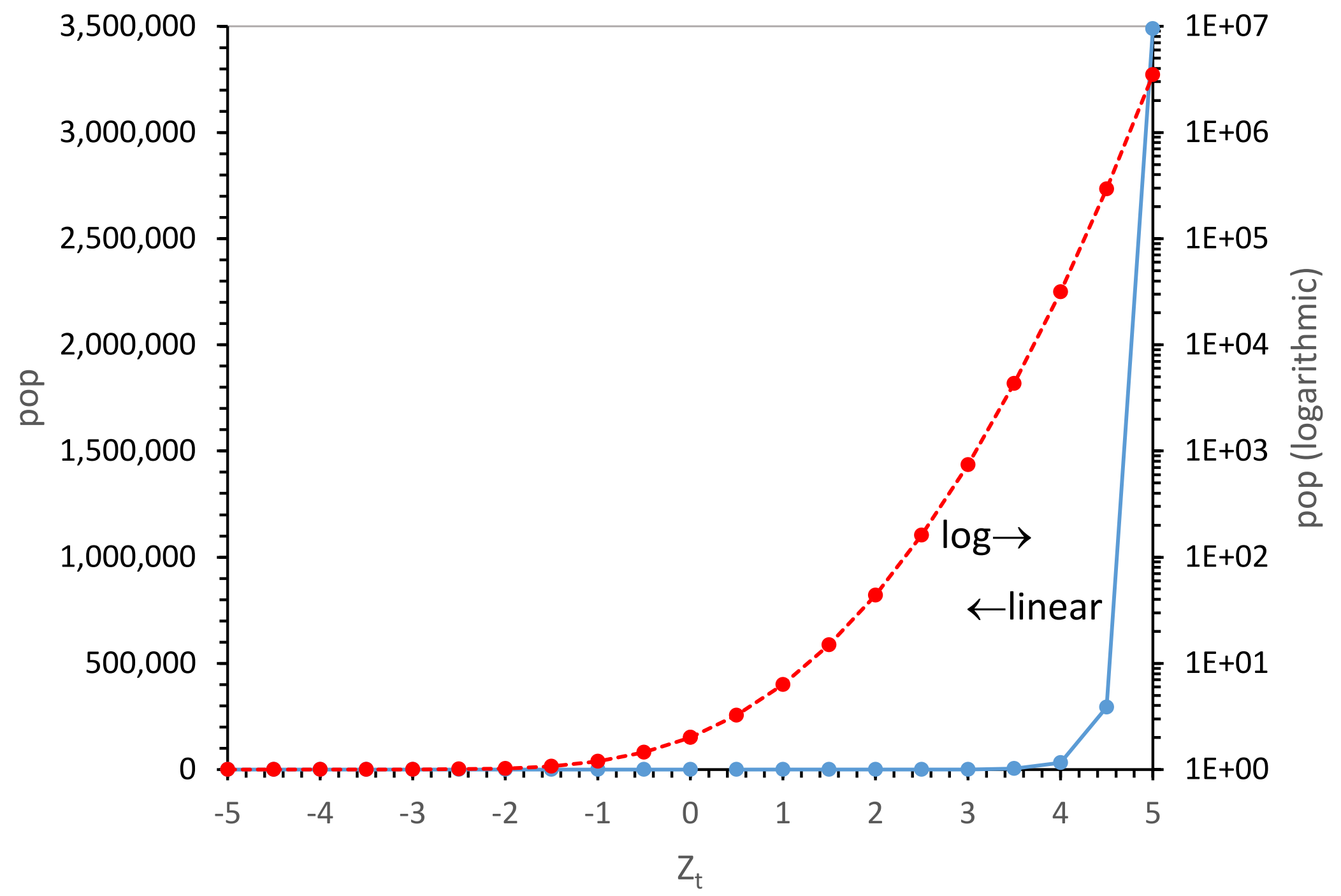

Figure 4A 


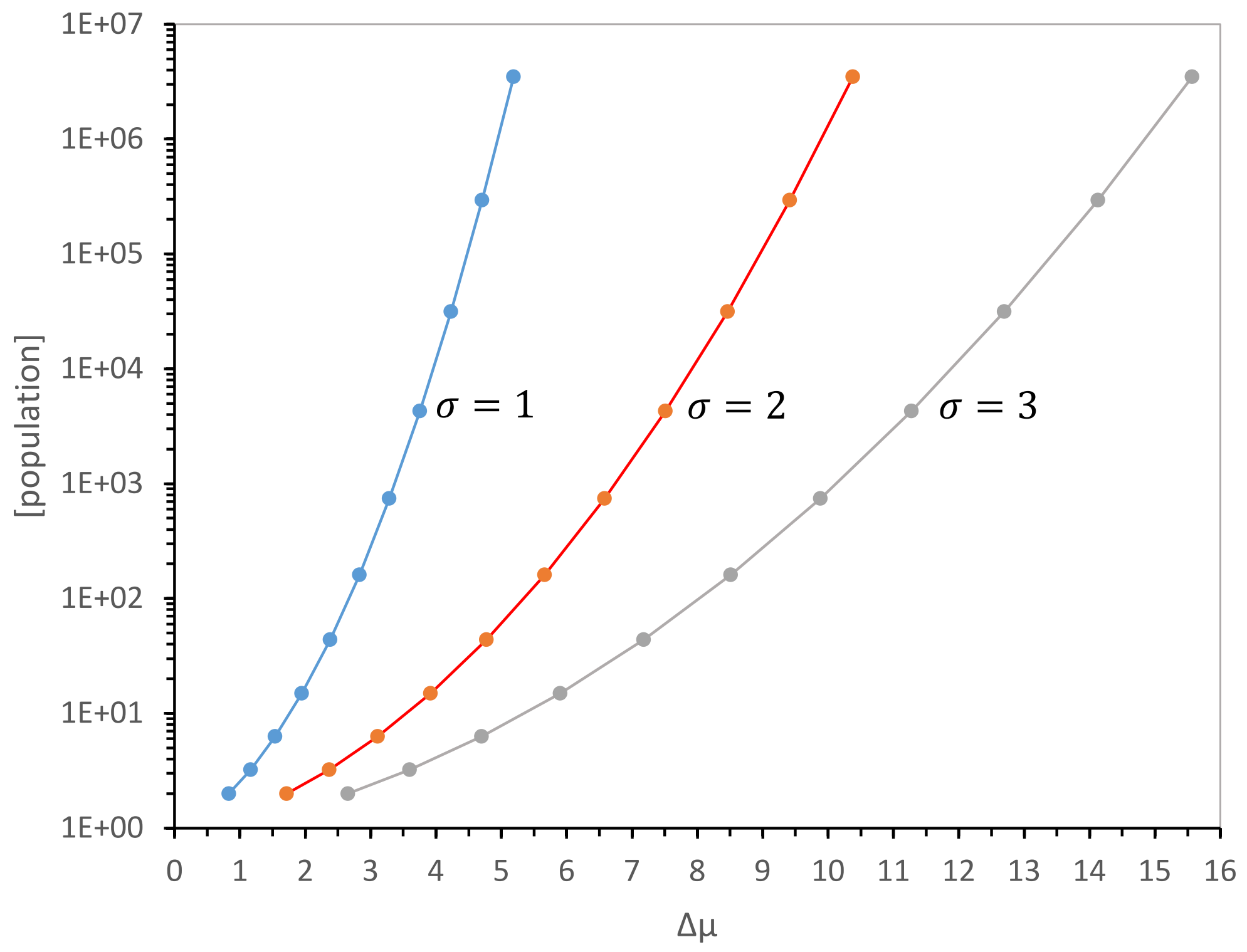

Figure 4B 


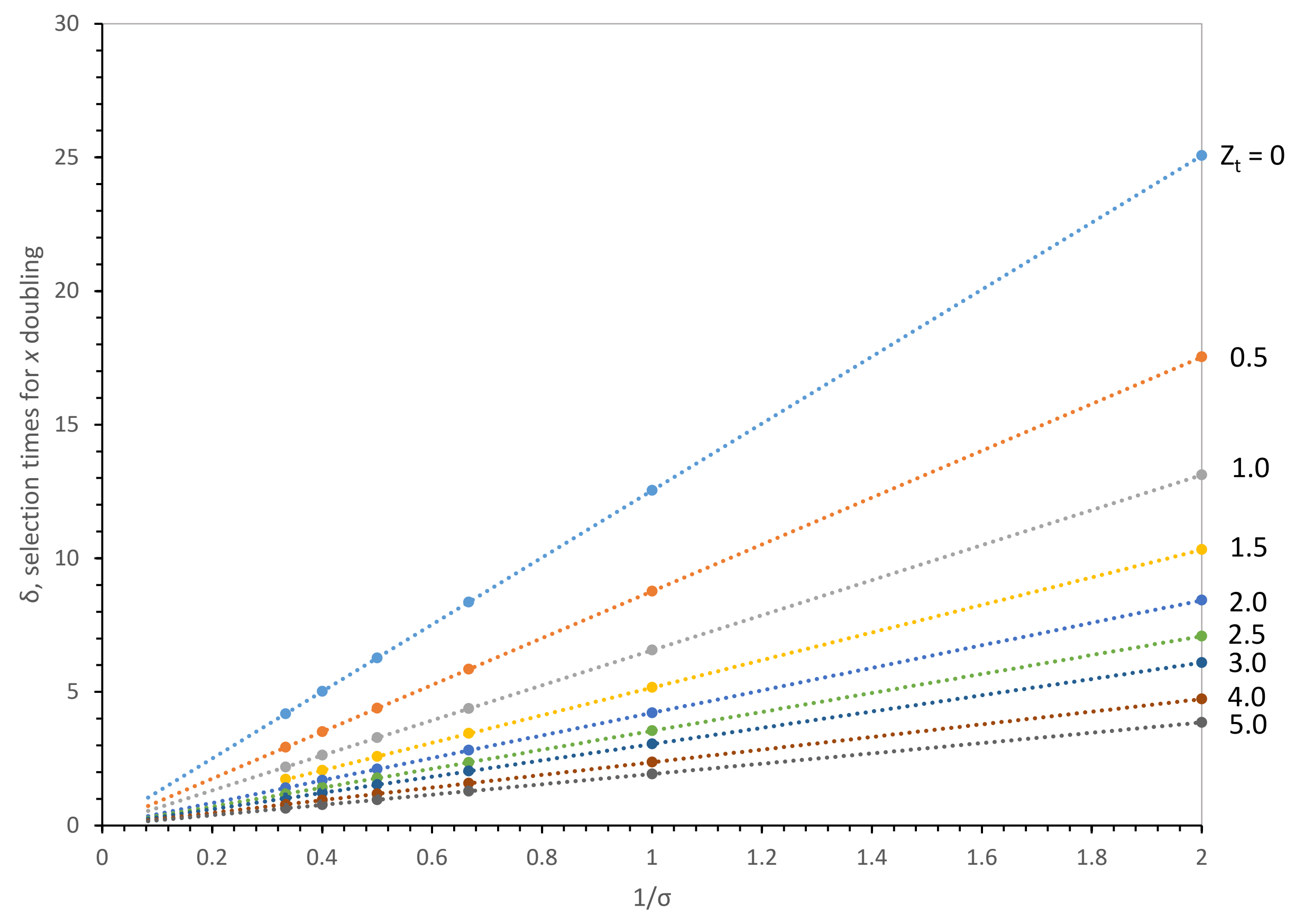

Figure 5 